where $\partial F / \partial \alpha$ and $\partial F / \partial R$ are found by using $0.01 \%$ changes in $\alpha$ and $R$. Convergence to the exact values is quite rapid requiring only three or four passes. Using a CDC 3600 computer where each pass required $8 \mathrm{sec}$, a neutral stability curve was found in approximately $10 \mathrm{~min}$ if initial guesses were made carefully.

\section{RESULTS AND CONCLUSIONS}

The velocity profiles corresponding to an imposed wall temperature difference of from $\Delta T=0^{\circ} \mathrm{F}$ to $\Delta T=$ $200^{\circ} \mathrm{F}$ are shown in Fig. 1. Since the viscosity of water decreases with increasing temperature, the resultant velocity profiles became skewed as $|\Delta T|$ increases; the maximum velocity is shifted toward the hotter wall.

Figure 2 shows that if the viscosity gradient terms are neglected in the stability analysis, the resultant neutral stability curve is shifted to the right as $|\Delta T|$ increases, thus indicating a more stable flow situation. This agrees with the results of Potter and Smith. ${ }^{2}$ In contrast, when the terms are accounted for, the flow becomes destabilized as $|\Delta T|$ increases which is the same effect noted by Wazzan et al..$^{5} \mathrm{It}$ should also be noted that a double critical point did not appear in the neutral stability curve when the viscosity gradient terms were included in the equation. However, neglecting the viscous terms led to the appearance of an inflection point (or possibly a double critical point for other $\Delta T^{\prime} \mathrm{s}$ ) at a Reynolds number of approximately 32000 for $\Delta T=100^{\circ} \mathrm{F}$ (Fig. 2). Hence, even though the viscosity gradient terms are small, their inclusion is extremely important. The results of Potter and Smith ${ }^{2}$ and Mott and Joseph are thus concluded to be in error.

Figure 3 plots the variation. of wave speed with Reynolds number for various values of temperature difference $\Delta t$.

Figure 4 plots the variation of the critical Reynolds number with wall temperature difference $\Delta T$. It can be seen that the temperature difference between the plates has a definite destabilizing effect; there is a $50 \%$ reduction in critical Reynolds number resulting from a $140^{\circ} \mathrm{F}$ temperature difference.

The curves for $\Delta T=0^{\circ} \mathrm{F}$ (Fig. 2) represent isothermal Poiseuille flow and agree with the accepted results of Thomas. ${ }^{1}$ Eigenfunctions are also plotted in Fig. 5 for $\Delta T=0^{\circ} \mathrm{F}$ and for $\Delta T=200^{\circ} \mathrm{F}$.

${ }^{1}$ L. H. Thomas, Phys. Rev. 91, 780 (1953).

${ }^{2}$ M. C. Potter and M. C. Smith, Phys. Fluids 11, 2763 (1968)

${ }^{3}$ J. E. Mott and D. D. Joseph, Phys. Fluids 11, 2065 (1967).

$4 \mathrm{G}$. Poots and M. H. Rogers, Intern. J. Heat Mass Transfer 8, 1515 (1965).

5 A. R. Wazzan, T. T. Okamura, and A. M. O. Smith, J. Heat Transfer (Trans. ASME) 90, $109(1960)$.

${ }^{6} \mathrm{~W}$. Heisenberg, Ann. Physik. (Leipzig) 74, 577 (1924).

7 C. C. Lin, Quart. Appl. Math. 3, 117, 218, 277 (1945).

${ }^{8}$ R. E. Kaplan, Ph.D. thesis, Massachusetts Institute of Technology (1964).

${ }^{9}$ W. C. Reynolds and M. C. Potter, J. Fluid Mech. 27, 465 (1967).

\title{
Ion-Temperature-Sensitive Effect in Transient Langmuir Probe Response
}

\author{
JUAN R. SANMARTIN \\ Department of Mechanical Nugineering, Massachusetts Institute of Technology, Cambridge, Massachusells 02139
} (Received 24 February 1971; final manuscript received 6 July 1971)

\begin{abstract}
A theory is presented for a method, recently proposed by Hester and Sonin, of determining the ion temperature in a plasma by measuring the transient current to a cylindrical Langmuir probe after applying a potential $V_{p}\left(-e V_{p} \gg_{K} T_{e}\right)$ under conditions where collection is collision free and the ratio of probe radius to Debye length is small. The ion component of the current does not approach its final steady-state value monotonically, but exhibits a strong, ion-temperature-dependent overshoot in the first few ion-plasma periods following the biasing of the probe. Analytical formulas are derived for the case of a Maxwellian plasma, and convenient graphical results are presented. The possible masking of the overshoot by a transient displacement current is discussed; it is shown how to avoid such displacement effects. For the overshoot to be sensitive to the ion temperature $T_{1}$, the probe must be near plasma (zero) potential before applying $Y_{p}\left(e \gamma_{p}-<0.1 \kappa T_{e}, \mathrm{~T}_{p}^{-}\right.$being that initial potential); this is not a drawback of the method, but, on the contrary, it can be used to accurately determine plasma potential along with $T_{2}$.
\end{abstract}

\section{INTRODUCTION}

Hester and Sonin ${ }^{1}$ have recently proposed a method of determining the ion temperature in a plasma by measuring the transient current drawn by a long, cylindrical Langmuir probe to which a large, negative potential has been applied stepwise. They performed some computations showing that the ion current ex- hibits an initial overshoot which is very sensitive to the ion temperature when the probe radius is small compared with the Debye length. Such an effect would be of considerable interest for diagnostic purposes because steady-state probe response is, under all conditions, very weakly dependent on that plasma property. ${ }^{2}$ 
In this paper, we present a theoretical formulation of that problem which yields both a simple analytical formula for the transient current and a clear understanding of the overshoot. The treatment is limited to the first ion-plasma period following the biasing of the probe, which is the region where the response is most sensitive to the ion temperature. The basic approach is discussed in the next section. In Sec. III we derive some needed results about the potential field set up by the probe. The qualitative behavior of the ion current is discussed in Sec. IV, where quantitative, final formulas are also presented (details of the derivation are given in the Appendix). The reader only interested in the applications may go directly to Sec. V, where the results are resumed and a detailed discussion of the usefulness and experimental limitations of the method is given. In particular, the possible masking of the overshoot by the displacement current, which is registered in the external measuring circuitry together with the ion current, is discussed. It is shown how to make the influence of the displacement current negligible. Also, an alternative, pulsed probe method is suggested in which displacement effects cancel out.

\section{BASIC FORMULATION}

Consider the problem of a quiescent plasma and an infinitely long, cylindrical Langmuir probe of radius $r_{p}$ to which a negative potential $V_{p}$ (relative to the plasma) is applied at $t=0$. It is desired to obtain the ion current to the probe for positive times. The potential field $V$ is governed by Poisson's equation, which in nondimensional form is

$$
\rho^{-1} \frac{\partial}{\partial \rho} \rho \frac{\partial}{\partial \rho} \psi=\epsilon^{2}\left(n_{i}-n_{e}\right),
$$

with boundary conditions

$$
\psi(\rho=1)=\psi_{p} \equiv-e V_{p} / k T_{e}, \quad \psi(\infty)=0,
$$

where

$$
\begin{gathered}
\psi=-e V / \kappa T_{e}, \quad \rho=r / r_{p}, \\
\epsilon=r_{p} / \lambda_{D}, \quad n_{i}=Z_{i} N_{i} / N_{0}, \quad n_{e}=N_{e} / N_{0} ;
\end{gathered}
$$

$N_{e}$ and $N_{i}$ are the electron and ion densities, $T_{\boldsymbol{e}}$ and $N_{0}$ the unperturbed electron temperature and density, $r$ is the radial distance to the probe axis, $Z_{i}$ is the ion charge number and $\lambda_{D}$ is the electron Debye length $\left(\kappa T_{e} / 4 \pi e^{2} N_{0}\right)^{1 / 2}$. Here it will be assumed that all mean free paths are large compared with $\lambda_{D}$, so that if $\epsilon \leq O(1)$, current collection will be collisionfree and $n_{s}$ and $n_{i}$ will be given by the corresponding time-dependent Vlasov equation. Simple dimensional considerations then show that

$$
j(\tau \geq 0) / j_{\infty}=\left(j / j_{\infty}\right)\left[\tau, \epsilon, \psi_{p}, \beta,\left(m_{e} / m_{i}\right)\right],
$$

where

$$
\beta=T_{i} / Z_{i} T_{e}, \quad \tau=\omega_{p i} i
$$

$j$ is the ion current density, $j_{\infty}$ is the steady-state current density corresponding to the probe potential $V_{p}, \omega_{p i}$ is the ion-plasma frequency $\left(4 \pi e^{2} Z_{i} N_{0} / m_{i}\right)^{1 / 2}$, $m_{e}$ is the electron mass, and $m_{i}$ and $T_{i}$ are the ion mass and temperature, respectively; Eq. (2) will also depend on the probe potential for negative times, if that is not zero. One expects $j / j_{\infty} \rightarrow 1$ as $\tau \rightarrow \infty$.

The general solution of the problem defined above is quite difficult. We are interested, however, in specific conditions under which $j / j_{\infty}$ does not increase monotonically from $j(\tau=0) / j_{\infty}$ to 1 , but exhibits a strong overshoot. It is quite fortunate that such conditions also result in a considerable simplification of the problem. We assume the following:

(a) The probe is at plasma potential at $\tau<0$. The selection of any other initial probe potential would somewhat mask the influence of $T_{i}$ on the overshoot, since this influence depends on the initial distribution function in the neighborhood of the probe. (This point will be discussed at length in Sec. V.) Then, the initial plasma state is ${ }^{2}$

$$
\begin{gathered}
\psi(\rho, \tau<0)=0 \\
n_{e}(\rho, \tau \leq 0)=n_{i}(\rho, \tau \leq 0)=1-\pi^{-1} \sin ^{-1} \rho^{-1}, \\
j(\tau \leq 0)=e N_{0}(2 \pi)^{-1 / 2}\left(\kappa T_{i} / m_{i}\right)^{1 / 2} .
\end{gathered}
$$

(b) $\psi_{p} \gg 1$. This assumption allows us to neglect the electron current. Moreover, it leads to a simple expression for $n_{e}$

$$
n_{e}=\exp (-\psi),
$$

valid everywhere except in the neighborhood of the probe, where $n_{e}$ will be exponentially small anyway [see also condition (d) below]. The equilibrium with the field will be reached in a few electron-plasma periods, so that the dependence of (2) on $m_{e} / m_{i}$ will disappear for, say, $r>0.1$.

(c) $\psi_{p} \gg \beta$, and (d) $\epsilon \ll 1$. These two conditions are found to be essential for the appearance of a large overshoot. Condition (d) also implies that steadystate current collection will be orbital motion limited, so that ${ }^{2}$

$$
j_{\infty}=e N_{0} 2^{1 / 2} \pi^{-1}\left(-Z_{i} e V_{p} / m_{i}\right)^{1 / 2},
$$

and, assuming the unperturbed plasma to be Maxwellian, ${ }^{2}$

$$
\begin{aligned}
n_{i}(\tau \rightarrow \infty) \rightarrow 1-\pi^{-1} \int_{0}^{\infty} d s & \exp (-s) \\
& \times \sin ^{-1}\left[\rho^{-1}\left(\frac{\psi_{p}+\beta s}{\psi+\beta s}\right)^{1 / 2}\right] .
\end{aligned}
$$

Furthermore, (d) greatly simplifies the determination of $\psi$, and also contributes to the validity of Eq. (5), since a region of order of $r_{p}$, where Eq. (5) fails, then contains a negligible amount of negative charge even if $n_{e}=O(1)$. 
All these conditions greatly simplify the analysis. First, it is possible to derive an accurate analytical expression for $\partial \psi / \partial \rho$ without simultaneously solving the ion Vlasov equation. That expression is only valid in a certain (large) neighborhood of the probe. This restricts the theory to times bound by an upper value $\tau_{m}$, since for sufficiently long times the probe will be collecting ions that were initially outside that neighborhood. Fortunately, again, the overshoot occurs at shorter times, and is, in fact, most sensitive to $T_{i}$ in the $\tau$ range where the theory is valid.

Second, once the field is known ion trajectories may be determined explicitly. Moreover, the current to the probe is now linear in the initial ion distribution function and this allows a further simplification. Let $f_{i}$ be the ion distribution function after integration over the velocity parallel to the probe. We shall assume that far away from the probe $f_{i}$ is isotropic:

$$
f_{i}(\rho \rightarrow \infty)=Z_{i}^{-1} N_{0} g\left(\nu_{\perp}\right),
$$

where $v_{\perp}$ is the ion speed in the $\rho$ - $\phi$ plane ( $\phi$ being a polar angle). $f_{i}$ is normalized such that

$$
\int_{0}^{\infty} 2 \pi v_{\perp} g\left(v_{\perp}\right) d v_{\perp}=1
$$

for a Maxwellian plasma,

$$
g=\left(m_{i} / 2 \pi \kappa T_{i}\right) \exp \left(-m_{i} v_{\perp}^{2} / 2 \kappa T_{i}\right) .
$$

Then, we clearly have

$$
\begin{aligned}
f_{i}(\tau \leq 0) & =Z_{i}^{-1} N_{0} g\left(v_{\perp}\right), & & |\gamma|>\sin ^{-1} \rho^{-1}, \\
& =0, & & |\gamma|<\sin ^{-1} \rho^{-1},
\end{aligned}
$$

where $\gamma$ is a velocity-vector angle $(-\pi \leq \gamma \leq \pi)$; this is illustrated in Fig. 1. For any $g$, Eqs. (9) result in the initial density given by $\mathrm{Eq}$. (3); the ion depletion is due to the particle loss caused by thermal motion. Now let $j^{*}$ be the current density obtained by assuming an initial ion distribution function with all ions having velocities with the same direction and magnitude, $v_{\perp} *$; see Fig. 2, where the polar axis has been chosen parallel to the velocity for simplicity. (We note that in the shaded area there are no ions

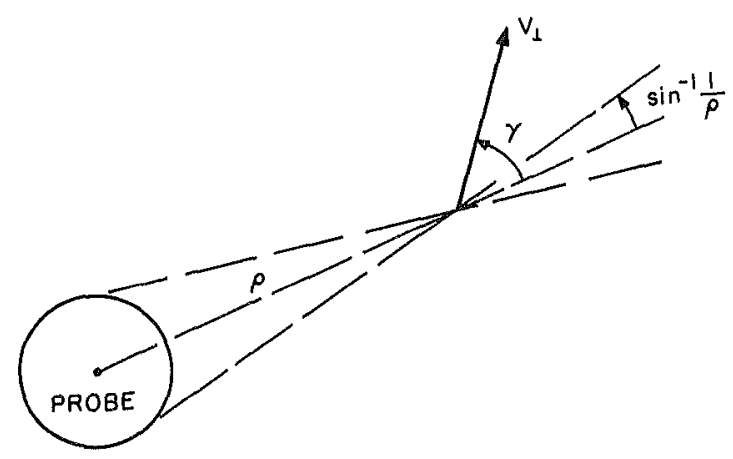

FIG. 1. Diagram for initial ion distribution function.

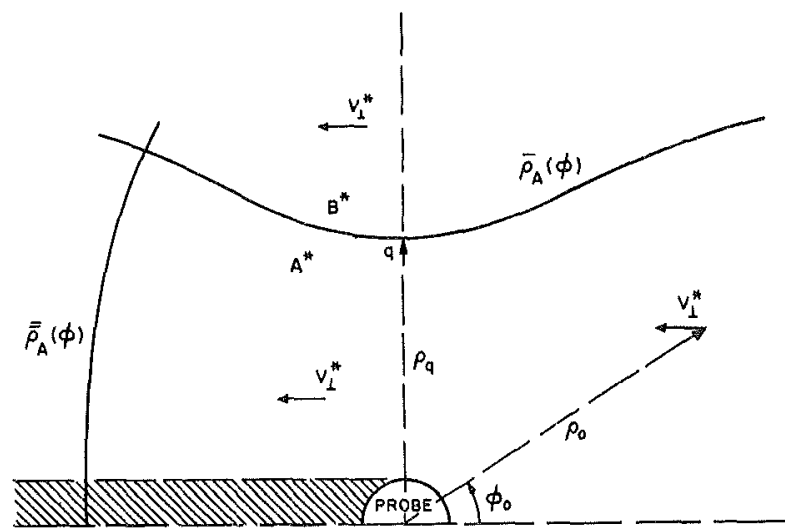

Fig. 2. Diagram showing regions $A^{*}$ and $B^{*}$ for simplest initial ion distribution function; density is zero in shaded region, $N_{0} / Z_{i}$ outside it. $A^{*}$ is the set of initial positions of ions that will be collected at positive times.

while outside it the density is uniform, $N_{0} / Z_{i}$.) Then, one easily concludes from the symmetry of the problem that $j$ may be written as

$$
j(\tau \geq 0)=\int_{0}^{\infty} 2 \pi v_{\perp} * g\left(v_{\perp}^{*}\right) d v_{\perp}^{*} j^{*}\left(v_{\perp}^{*}, \tau \geq 0\right) .
$$

Finally, the determination of $j^{*}$ itself may be simplified by using the following approach. The nonshaded region of Fig. 2 may be divided in two mutually exclusive parts $A^{*}$ and $B^{*}$. A point $\rho-\phi$ belongs to $A^{*}$ if an ion having such initial coordinates will hit the probe at some time $\tau>0$. Computing the time of flight of an $A^{*}$ ion to the probe we may then define a function $a^{*}(\tau)$ that represents the area of region $A^{*}$ that has been "collected" by the time $\tau$. From the uniformity of the density it follows that

$$
2 \pi r_{p} j^{*}=e N_{0} d a^{*} / d t
$$

or

$$
\frac{j^{*}}{j_{\infty}}=\frac{\epsilon}{\left(8 \psi_{p}\right)^{1 / 2}} \frac{d a^{*}}{d \tau}
$$

where $\hat{a}^{*}$ is a nondimensional area, $\hat{a}^{*}=a^{*} / r_{p}{ }^{2}$. For the time-averaged current we have

$$
\frac{\bar{j}^{*}}{j_{\infty}} \equiv \tau^{-1} \int_{0}^{\tau} \frac{j^{*}\left(\tau^{\prime}\right)}{j_{\infty}} d \tau^{\prime}=\frac{\epsilon \hat{d}^{*}}{\left(8 \psi_{p}\right)^{1 / 2} \tau}
$$

In the next section we will find the aforementioned expression for the electric field $\partial \psi / \partial \rho$. Subsequently, qualitative (Sec. IV) and quantitative (appendix) derivations of $a^{*}(\tau)$ will be given. Once $\hat{a}^{*}(\tau)$ is known, both $j(\tau)$ and $j(r)$ follow immediately from Eqs. (10) and (11).

\section{THE POTENTIAL FIELD}

To compute $\hat{a}^{*}(\tau)$ we shall have to integrate the electric field $\partial \psi / \partial \rho$ along the trajectory of a particle to obtain its velocity and its time of flight to the 
probe. A formal expression for $\partial \psi / \partial \rho$ can be obtained from $\mathrm{Eq}$. (1),

$$
\frac{\partial \psi}{\partial \rho}=\frac{-\psi_{p} \delta(\tau)}{\rho}+\frac{\epsilon^{2}}{\rho} \int_{1}^{\rho} \rho^{\prime} d \rho^{\prime} \nu\left(\rho^{\prime}, \tau\right)
$$

where

$$
\delta \equiv-\psi_{p}^{-1} \partial \psi /\left.\partial \rho\right|_{\rho=1}, \quad \nu \equiv n_{i}-n_{e}
$$

Eq. (12) may be rewritten as

$$
\frac{\partial \psi}{\partial \rho}=-\frac{\psi_{p} \delta(\tau)}{\rho}+\frac{\epsilon^{2}}{\rho} \frac{1}{2}\left(\rho^{2}-1\right)\langle\nu(\rho, \tau)\rangle,
$$

$\langle\nu\rangle$ being given by

$$
\left(\rho^{2}-1\right)\langle\nu\rangle=\int_{1}^{\rho 2} \nu d\left(\rho^{\prime 2}\right)
$$

We note that $\delta$ is proportional to the electric field at the probe. Its relation to the over-all charge density follows easily from (12):

$\delta=\psi_{p}^{-1} \epsilon^{2} \int_{1}^{\infty} \rho d \rho \nu=\left(\int_{1}^{\infty} \rho d \rho \nu\right)\left(\int_{1}^{\infty} \rho d \rho \nu \ln \rho\right)^{-1}$.

Thus, $\delta^{-1}$ represents the logarithm of a characteristic shielding distance.

To carry out the aforementioned integrations we shall substitute constants $\bar{\delta}$ and $\vec{\nu}$ for $\delta(\tau)$ and $\langle\nu(\rho, \tau)\rangle$ in Eq. (13). Our purpose is to show that $\delta$ and (with some restrictions on $\rho$ ) $\langle\nu\rangle$ vary little, and to find close bounds for them; this will allow us to choose appropriate values for $\vec{\delta}$ and $\vec{\nu}$, and to determine the error in the approximations used. The basic approach will be to study the limits $\tau \rightarrow 0$ and $\tau \rightarrow \infty$, for which $\nu$ is known explicitly [see Eqs. (3), (5), and (7)].

\section{A. Bounds for $\delta$ at $\tau \approx 0^{3}$ and $\tau \rightarrow \infty$}

We must first establish the following theorem: Let $\psi_{k}(\rho)$ be defined by

$$
\begin{gathered}
\rho^{-1} \frac{\partial}{\partial \rho} \rho \frac{\partial}{\partial \rho} \psi_{k}=F_{k}\left[\psi_{k}(\rho), \rho\right] \quad \rho \geq 1, \\
\psi_{k}(1)=\psi_{p}, \quad \psi_{k}(\infty)=0,
\end{gathered}
$$

where $k=1,2$, and $F_{k}$ is bounded, compatible with Eqs. (17), and such that $\psi_{k}$ is continuous and monotonic. Let

$$
F_{2}[f, \rho] \geq F_{1}[f, \rho] \quad \rho \geq 1, \quad \psi_{p} \geq f \geq 0,
$$

and $F_{1}[f, \rho]$ be nondecreasing in $f$. Then, $\delta_{2} \geq \delta_{1}$ if

$$
\delta_{k} \equiv-\psi_{p}{ }^{-1} \partial \psi_{k} /\left.\partial \rho\right|_{\rho=1} .
$$

To prove this let us assume that $\delta_{2}<\delta_{1}$. Integrating (16) twice we get

$$
\psi_{k}=\psi_{p}\left[1-\delta_{k} \ln \rho\right]+\int_{1}^{\rho} \rho^{\prime} d \rho^{\prime} \ln \frac{\rho}{\rho^{\prime}} F_{k}\left[\psi_{k}\left(\rho^{\prime}\right), \rho^{\prime}\right] .
$$

Thus in a certain neighborhood of 1 , we have $\psi_{2}>\psi_{1}$.
Let $s$ be the minimum value of $\rho$ such that $\psi_{2}(s)=$ $\psi_{1}(s)$; such a value exists, whether finite or infinite, because $\psi_{2}(\infty)=\psi_{1}(\infty)$. It follows that

$$
\begin{aligned}
& 0=\psi_{1}(s)-\psi_{2}(s)=\psi_{p}\left(\delta_{2}-\delta_{1}\right) \ln s+\int_{1}^{s} \rho d \rho \ln \frac{s}{\rho} \\
& \times\left\{F_{1}\left[\psi_{1}(\rho), \rho\right]-F_{2}\left[\psi_{2}(\rho), \rho\right]\right\} \leq \psi_{p}\left(\delta_{2}-\delta_{1}\right) \ln s \\
& \quad+\int_{1}^{s} \rho d \rho \ln \frac{s}{\rho}\left\{F_{1}\left[\psi_{1}(\rho), \rho\right]-F_{1}\left[\psi_{2}(\rho), \rho\right]\right\}<0
\end{aligned}
$$

proving that $\delta_{2}$ cannot be less than $\delta_{1}$.

Upper and lower bounds for $\delta(\tau \approx 0)$ can now be found. The right-hand side of Eq. (1) is then, according to Eqs. (3) and (5),

$$
F=\epsilon^{2}[1-\exp (-\psi)]-\left(\epsilon^{2} / \pi\right) \sin ^{-1} \rho^{-1} .
$$

Introducing

$$
\begin{aligned}
& F_{2}{ }^{(1)}\left[\psi_{2}{ }^{(1)}(\rho), \rho\right]=\epsilon^{2}\left[1-\exp \left(-\psi_{p}\right)\right]-\left(\epsilon^{2} / \pi\right) \sin ^{-1} \rho^{-1} \\
& \psi_{2}^{(1)} \geq 1-\exp \left(-\psi_{p}\right), \\
&=\epsilon^{2} \psi_{2}^{(1)}-\left(\epsilon^{2} / \pi\right) \sin ^{-1} \rho^{-1} \\
& \psi_{2}^{(1)} \leq 1-\exp \left(-\psi_{p}\right),
\end{aligned}
$$

our theorem can be seen to apply if $F \rightarrow F_{1}, \psi \rightarrow \psi_{1}$, and $F_{2}{ }^{(1)} \rightarrow F_{2}, \psi_{2}{ }^{(1)} \rightarrow \psi_{2}$; therefore, $\delta(\tau \approx 0) \leq \delta_{2}{ }^{(1)}$. For a lower bound, let us introduce

$$
\begin{aligned}
& F_{1}{ }^{(2)}\left[\psi_{1}^{(2)}(\rho), \rho\right]=\epsilon^{2}[1-\exp (-p)] \\
& -\left(\epsilon^{2} / \pi\right) \sin ^{-1} \rho^{-1}, \quad \psi_{1}{ }^{(2)} \geq p, \\
& =\epsilon^{2}\{[1-\exp (-p)] / p\} \psi_{1}{ }^{(2)} \\
& -\left(\epsilon^{2} / \pi\right) \sin ^{-1} \rho^{-1}, \quad \psi_{1}^{(2)} \leq p,
\end{aligned}
$$

where $p \equiv 1-\exp \left(-\psi_{p}\right)$. Our theorem again applies with $\quad F \rightarrow F_{2}, \quad \psi \rightarrow \psi_{2}$ and $F_{1}{ }^{(2)} \rightarrow F_{1}, \quad \psi_{1}{ }^{(2)} \rightarrow \psi_{1} ; \quad$ thus $\delta(\tau \approx 0) \geq \delta_{1}{ }^{(2)}$.

For $\delta(\tau \rightarrow \infty)$, we have [according to $\mathrm{Eq} .(7)]$

$$
\begin{aligned}
F=\epsilon^{2}[1-\exp (-\psi)]-\frac{\epsilon^{2}}{\pi} \int_{0}^{\infty} d s & \exp (-s) \\
& \times \sin ^{-1}\left[\rho^{-1}\left(\frac{\psi_{p}+\beta s}{\psi+\beta s}\right)^{1 / 2}\right]
\end{aligned}
$$

as the right-hand side of Eq. (1). An upper bound follows immediately: $\delta(\tau \rightarrow \infty) \leq \delta(\tau \approx 0) \leq \delta_{2}{ }^{(1)}$ since $\partial F / \partial \psi>0$ and

$$
\int_{0}^{\infty} d s \exp (-s) \sin ^{-1}\left[\rho^{-1}\left(\frac{\psi_{p}+\beta s}{\psi+\beta s}\right)^{1 / 2}\right] \geq \sin ^{-1} \rho^{-1}
$$

for $0 \leq \psi \leq \psi_{p}$. For a lower bound we note that if $\overline{\bar{\beta}}>\bar{\beta}$, then $\bar{\delta}(\tau \rightarrow \infty, \bar{\beta}) \geq \delta(\tau \rightarrow \infty, \bar{\beta})$ since $\left(\psi_{p}+\overline{\bar{\beta}} s\right) /(\psi+\overline{\bar{\beta}} s) \leq$ $\left(\psi_{p}+\bar{\beta} s\right) /(\psi+\bar{\beta} s)$ for $s>0$ and $0 \leq \psi \leq \psi_{p}$; thus, it suf- 
Frg. 3. Auxiliary function $Y$ vs $\psi_{p}$ and $\epsilon$. $Y$ is defined in Eq. (24).

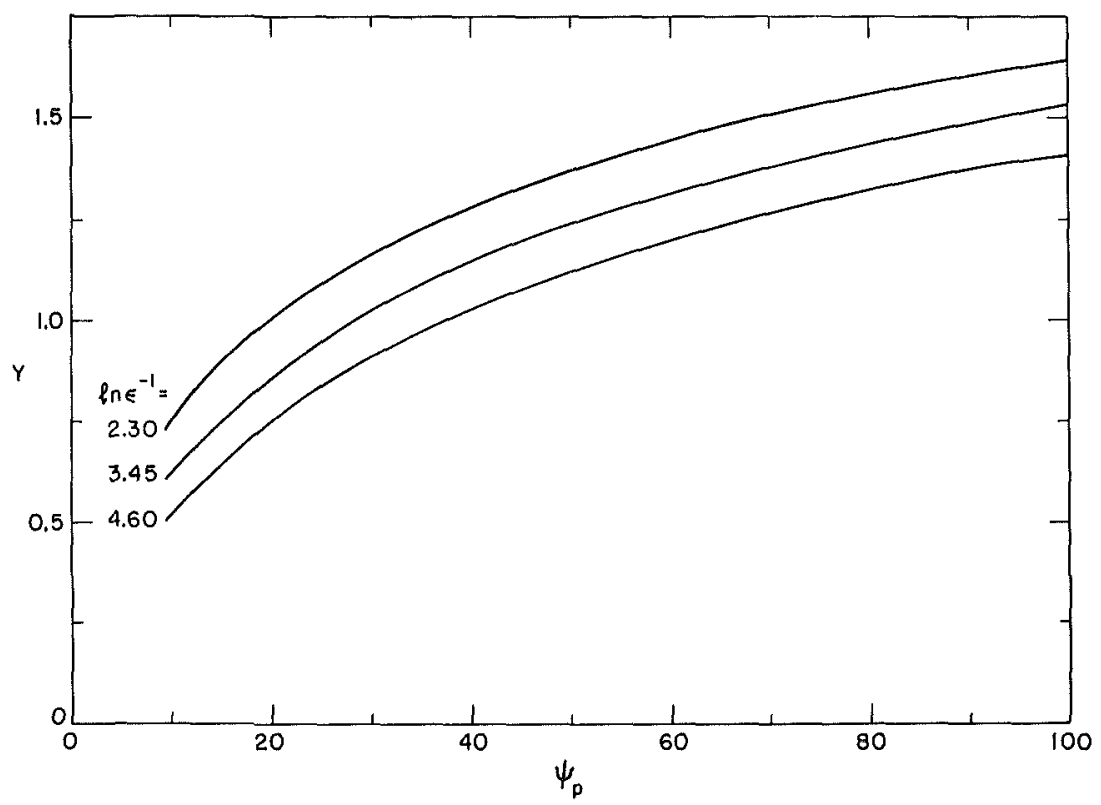

fices to find a lower bound for $\delta(\tau \rightarrow \infty, \beta \rightarrow 0)$. Let us introduce

$$
\begin{aligned}
& F_{1}^{(3)}=\epsilon^{2}[1-\exp (-p)]-\frac{\epsilon^{2}}{\pi} \int_{0}^{\infty} d s \exp (-s) \\
& \times\left\{\frac{1}{2} \pi, \sin ^{-1}\left[\rho^{-1}\left(\frac{\psi_{p}+\beta s}{p+\beta s}\right)^{1 / 2}\right]\right\}
\end{aligned}
$$$$
=\epsilon^{2} \frac{1-\exp (-p)}{p} \psi_{1}^{(3)}-\frac{\epsilon^{2}}{\pi} \int_{0}^{\infty} d s \exp (-s)
$$$$
\times\left\{\frac{1}{2} \pi, \sin ^{-1}\left[\rho^{-1}\left(\frac{\psi_{p}+\beta s}{\left(p \rho_{(3)}{ }^{2} / \rho^{2}\right)+\beta s}\right)^{1 / 2}\right]\right\} \quad \psi_{1}^{(3)} \leq p
$$

where again $p=1-\exp \left(-\psi_{p}\right)$, and in the brackets inside the integrals above, the second terms are to be used whenever the arguments of $\sin ^{-1}$ are less than 1 ; otherwise, $\pi / 2$ should be used. Notice that we have retained a finite $\beta$ : if $\beta \rightarrow 0$ in $(25), \psi_{1}{ }^{(3)}$ cannot be made to vanish at infinity. However, if we let $\beta \rightarrow 0$ after solving for $\psi_{1}{ }^{(3)}$ and $\delta_{1}{ }^{(3)}$, we find a finite $\delta_{1}{ }^{(3)}$; this peculiar behavior is related to the wellknown singularity of the cold-ion limit in the steady state theory of ion-attracting cylindrical probes. ${ }^{2}$ We also note that $F_{1}^{(3)}$ is not a point function but a functional of $\psi_{1}{ }^{(3)}$, because of the appearance in Eq. (25) of $\rho_{(3)}$, defined as the point where $\psi_{1}^{(3)}=p$; making $F \rightarrow F_{2}, \psi \rightarrow \psi_{2}$ and $F_{1}{ }^{(3)} \rightarrow F_{1}, \psi_{1}{ }^{(3)} \rightarrow \psi_{1}$, one can see that (18) is nevertheless satisfied ${ }^{4}$ and that $F_{1}$, i.e., $F_{1}^{\left({ }^{(3)}\right.}$, satisfies a certain nondecreasing condition: if $\psi_{1}{ }^{(3)}\left(\rho^{\prime}\right) \leq$ $\psi\left(\rho^{\prime}\right)$ in the entire range $1 \leq \rho^{\prime} \leq \rho$, then $F_{1}^{(3)}\left[\psi_{1}^{(3)}, \rho\right] \leq$ $F_{1}{ }^{(3)}[\psi, \rho]$. Accounting for this, our theorem may be seen to apply again, giving $\delta(\tau \rightarrow \infty, \beta) \geq \delta \quad(\tau \rightarrow \infty$, $\beta \rightarrow 0) \geq \delta_{1}{ }^{(3)}$.

\section{B. Bounds for $\langle\nu\rangle$ at $\tau \approx 0$ and $\tau \rightarrow \infty$}

For $\tau \approx 0$, we have $\nu=1-\pi^{-1} \sin ^{-1} \rho^{-1}-\exp (-\psi)$. Thus, $\nu(1) \approx \frac{1}{2}$, and as $\rho$ increases, $\nu$ rapidly approaches unity and remains close to it until the electron density becomes important. Since space charge does not affect the field close to the probe, it is clear that in a certain (large) neighborhood of the probe $\langle\nu\rangle$ can be well approximated by a constant close to unity.

A natural limit to this region can be obtained by substituting a constant $\bar{\delta}$ for $\delta(\tau)$ [see Eq. (24) below] in (13) and defining

$$
\rho_{m}=\left(2 \psi_{p} \bar{\delta}\right)^{1 / 2} \epsilon^{-1} .
$$

Since $\rho_{m}^{2} \gg 1$ and $\partial \psi / \partial \rho$ should be negative, we must have $\langle\nu\rangle \rho^{2} / \rho_{m}{ }^{2}<1$ so that for $\rho>\rho_{m}$, $\langle\nu\rangle$ decays rapidly. Using $\psi_{2}^{(1)}$ and $\psi_{1}^{(2)}$, as given by Eqs. (16), (17), (19), and (20), as approximations for $\psi(\tau \approx 0)$, we find that $\langle\nu\rangle$ at $\rho=\rho_{m}$ is always larger than 0.60 . We have therefore $0.60<\langle\nu\rangle<1$ for $1 \leq \rho \lesssim \rho_{m}$ (by $\rho \lesssim \rho_{m}$ we mean that $\rho$ can be slightly larger than $\rho_{m}$; typically, $\left.\rho \leq 1.1 \rho_{m}\right)$.

Equations (3) and (7) show that $n_{i}(\tau \rightarrow \infty)<$ $n_{i}(\tau \approx 0)$. On the other hand, $n_{e}(\tau \rightarrow \infty)<n_{e}(\tau \approx 0)$ too, since $\delta(\tau \rightarrow \infty)<\delta(\tau \approx 0)$. Using $\psi_{1}^{(3)}$ as an approximation for $\psi(\tau \rightarrow \infty)$ it can be verified that $0.60<$ $\langle\nu\rangle<1$ in the range $1 \leq \rho \lesssim \rho_{m}$ for $\tau \rightarrow \infty$.

The condition $\rho \lesssim \rho_{m}$ will appear again in our analysis. We may consider $\rho_{m}$ as a sheath radius since $\ln \rho_{m}$ is always close to $\bar{\delta}^{-1}$, and moreover both the charge density and the electric field decay rapidly for $\rho>\rho_{m}$. It may be illustrative to note that assuming an idealized charge density $\nu=1$ up to a certain distance $\rho_{F}$ and $\nu=0$ for $\rho>\rho_{F}$, the left equality on Eq. (15) gives $\rho_{F}=\rho_{m}$. The right equality then yields 
$\bar{\delta}=\left(\ln \rho_{m}-\frac{1}{2}\right)^{-1}$; this may be used as an approximate equation for $\bar{\delta}$

$$
2 \bar{\delta}^{-1}=\ln \psi_{p}^{\prime}+\ln \bar{\delta}-2 \ln \epsilon+\ln 2-1 .
$$

\section{Bounds for Arbitrary $\tau$}

According to Eqs. (3) and (7) there is a net de crease in the ion density around the probe, between $\tau=0$ and $\tau=\infty$. Let us assume that this ion depletion is monotonic in time. Then, $\delta(\tau \approx 0) \geq \delta(\tau) \geq$ $\delta(\tau \rightarrow \infty)$ since $\delta$ goes down with the ion density. It also follows that $0.60<\langle\nu\rangle<1$ for $1 \leq \rho \lesssim \rho_{m}$ for all $\tau$. Actually, the preceding conclusions would hold under the weaker condition $n_{i}(\tau \approx 0) \geq n_{i}(\tau) \geq n_{i}(\tau \rightarrow \infty)$. In fact, since both $\delta$ and $\langle\nu\rangle$ depend on $n_{i}$ in a global way only, even this weaker condition could be locally violated without invalidating the general conclusions on $\delta$ and $\langle\nu\rangle$. The assumption $\partial n_{i} / \partial \tau<0$ appears to be well justified since the current density $j(\tau)$ ex ceeds $j_{\infty}$ quite soon and remains that way thereafter.

We are now in a position to write an approximation to Eq. (13). We define $1 / \bar{\delta}$ as $\left(1 / 2 \delta_{2}{ }^{(1)}\right)+\left(1 / 2 \delta_{1}{ }^{(3)}\right)$ This can be rewritten as

$$
1 / \bar{\delta}=\ln \epsilon^{-1}+Y
$$

where the function $Y\left(\epsilon, \psi_{p}\right)$ has been calculated and is given in Fig. 3. We also define

$$
\vec{\nu}=0.80 \text {. }
$$

Then, we approximate Eq. (13) as

$$
\frac{\partial \psi}{\partial \rho}=\frac{-\psi_{p} \bar{\delta}}{\rho}\left(1-\bar{\nu} \frac{\rho^{2}}{\rho_{m}^{2}}\right), \quad 1 \leq \rho \lesssim \rho_{m} .
$$

All three $\delta_{1}{ }^{(2)}, \delta_{2}{ }^{(1)}$, and $\delta_{1}{ }^{\left({ }^{3}\right)}$ are very close to each other so that the error in equating $\delta(\tau)$ to $\bar{\delta}$ is very small. The error goes down with increasing $\psi_{p}$ and $\epsilon^{-1}$ : for $\psi_{p}=\epsilon^{-1}=10$ the error is less than $5 \%$ and for $\epsilon^{-1}=10^{2}, \psi_{p} \geq 10$, it is less than $1 \%$. This estimate is conservative since the lower bound for $\delta$ was obtained from the limit $\tau \rightarrow \infty$ while only limited values of $\tau$ will be studied. Moreover, $\partial \psi / \partial \rho$ will only be used under integral signs, leading to smaller effective errors.

The approximation $\langle\nu\rangle=\bar{\nu}$ has a maximum possible error of \pm 0.20 . We again note that the integrations to be performed on $\partial \psi / \partial \rho$ should reduce the error (typically to \pm 0.10 ). The influence that the use of Eq. (26) may have in the determination of the ion temperature will be discussed in Sec. V.

\section{ION CURRENT BEHAVIOR}

The general behavior of $\bar{j} / j_{\infty}$ can be determined from a qualitative discussion of the function $\bar{j}^{*} / j_{\infty}$, by setting $v_{\perp}^{*}$ equal to the ion thermal velocity. Since angular momentum is conserved, the equation for the radial motion of an ion can immediately be integrated by using Eq. (26); in nondimensional form we find

$$
\begin{aligned}
\frac{1}{2} \epsilon^{2}\left(\frac{d \rho}{d \tau}\right)^{2}=-\psi_{p} \bar{\delta} \ln \frac{\rho}{\rho_{0}}+\beta^{*}\left(1-\frac{\rho_{0}^{2}}{\rho^{2}}\right. & \left.\sin ^{2} \phi_{0}\right) \\
& -\frac{1}{4} \bar{\nu} \epsilon^{2}\left(\rho_{0}^{2}-\rho^{2}\right),
\end{aligned}
$$

where $r_{0}\left(\equiv r_{p} \rho_{0}\right)$ and $\phi_{0}$ are the initial coordinates of the ion, and

$$
\beta^{*} \equiv m_{i}\left(v_{\perp}\right)^{2} / 2 Z_{i} \kappa T_{e} .
$$

For $\left(\rho_{0}, \phi_{0}\right)$ to belong to $A^{*}$ (Fig. 2) the right-hand side of Eq. (27) must be nonnegative at $\rho=1$. This leads to

$$
\left|\sin \phi_{0}\right| \leq G\left(\rho_{0}\right) / \rho_{0}
$$

$G \equiv\left[1+\left(\alpha^{*}\right)^{-1} \ln \rho_{0}^{2}-\bar{\nu}\left(\rho_{0}^{2}-1\right) / \alpha^{*} \rho_{m}^{2}\right]^{1 / 2}, \quad \alpha^{*} \equiv 2 \beta^{*} / \psi \hat{\delta}$.

For $\alpha^{*}<1$ there is a value $\rho_{q}>1$ such that $G\left(\rho_{q}\right)=$ $\rho_{q}$; then, $G\left(\rho_{0}\right) / \rho_{0}>1$ for $\rho_{0}<\rho_{q}$. As shown below the regime of interest is $\alpha^{*} \ll 1$; condition (c) (Sec. II) insures that such condition is satisfied in our analysis. The boundary of $A^{*}$ given by the equal sign in (29), and denoted by $\bar{\rho}_{A}(\phi)$, then has the shape schematically represented in Fig. 2. Now, for, say, $\rho<\rho_{m} / 3$ we have $\partial \psi / \partial \rho \propto \rho^{-1}$ [see Eq. (26)], and it may be shown that if $\rho_{0}$ is in that region, the mean velocity of an ion on its trip to the probe is nearly independent of $\rho_{0}$; specifically, one finds $\rho_{0} / \tau_{0} \approx \rho_{m}$, where $\tau_{0}\left(\rho_{0}, \phi_{0}\right)$ is the time of flight to the probe. Therefore, (as long as $\left.\rho_{0}<\rho_{q}\right)$ we have $\hat{a}^{*}(\tau) \approx \rho_{m}{ }^{2} \tau^{2} \propto \psi_{p} \bar{\delta} \tau^{2} / \epsilon^{2}$ and

$$
\bar{\jmath}^{*} / j_{\infty} \propto \epsilon \hat{a}^{*} / \tau \psi_{p}{ }^{1 / 2} \propto \psi_{p}{ }^{1 / 2} \tau \bar{\delta} / \epsilon .
$$

The function $\bar{\jmath}^{*} / j_{\infty}$ grows linearly with $\tau$ until $\tau=\tau_{q} \equiv$ $\tau_{0}\left(\rho_{0}=\rho_{q}, \phi_{0}=\pi / 2\right)$, at which point the dependence of $\hat{a}^{*}$ on $\tau$, originally quadratic, becomes nearly linear; for even larger $\tau$, ions from outside the $\partial \psi / \partial \rho \propto \rho^{-1}$ region will begin to be collected so that $\rho_{0} / \tau_{0}$ will decrease rapidly and therefore, $j^{*} / j_{\infty}$ will peak and begin to fall. (It is easy to verify that $\bar{j}^{*} / j_{\infty} \rightarrow 1$ as $\tau \rightarrow \infty$.) From (29) we get $\rho_{q} \approx\left(\psi_{p} \bar{\delta} / \beta^{*}\right)^{1 / 2}\left[\ln \left(\psi_{p} \bar{\delta} / \beta^{*}\right)\right]^{1 / 2}$ so that putting $\tau=\tau_{q} \approx \rho_{q} / \rho_{m}$ in Eq. (30) we get the peak current

$$
\bar{\jmath}^{*} / j_{\infty} \propto\left(\psi_{p} / \beta^{*}\right)^{1 / 2} \bar{\delta}\left[\ln \left(\psi_{p} \bar{\delta} / \beta^{*}\right)\right]^{1 / 2} .
$$

Equation (31) is fairly insensitive to the value of $\epsilon$; this prediction is supported by the numerical results of Ref. 1.

The peak can be far greater than unity and depends on $\beta^{*}$. This overshoot may be seen as caused by the sudden setup of the potential field which traps low angular momentum ions in the neighborhood of the probe (in the steady-state corresponding to a probe potential $\psi_{p}$ most of that neighborhood would be populated by high-angular momentum ions); when $\beta^{*}$ decreases, $\rho_{q}$ increases and the low angular momentum region increases too. 
FIG. 4. Auxiliary function $X$ vs $\eta$ and $\sigma$. Curves end at $2 \beta / \psi_{p} \bar{\delta} \approx 0.3 . X, \sigma$, and $\eta$ are defined in Eqs. (33), (35), and (36).

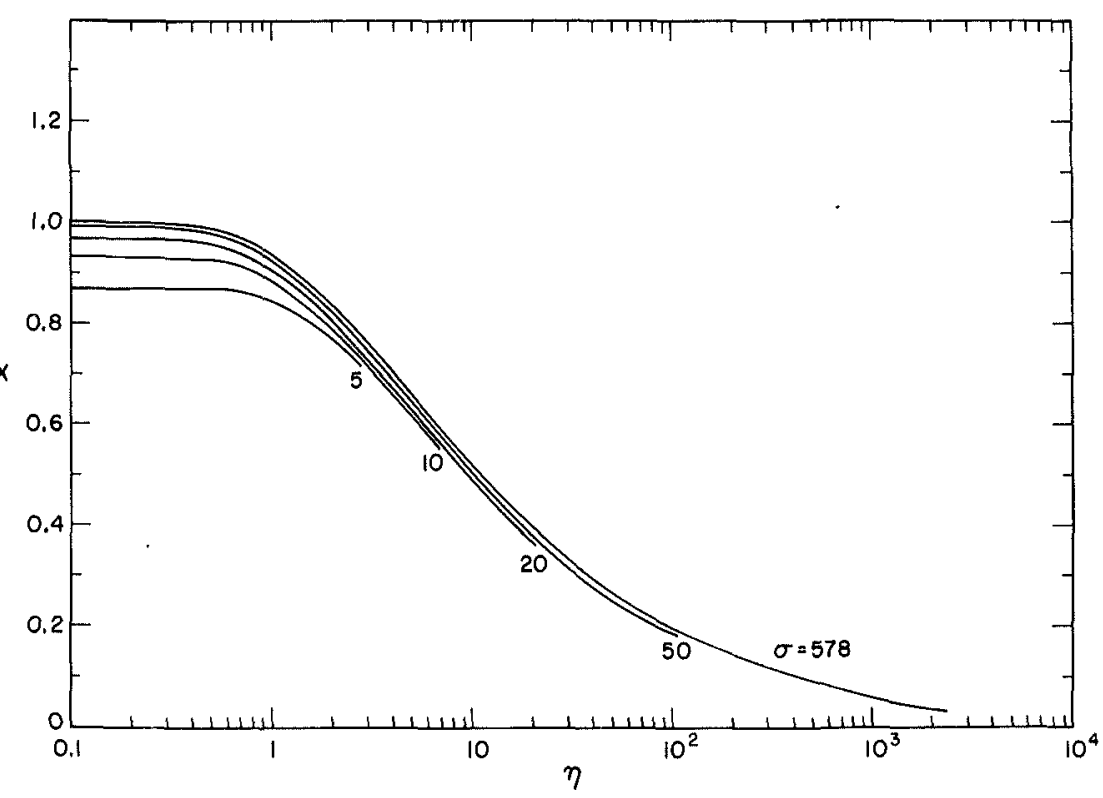

Eventually, however, a value of $\beta^{*}$ is reached for which $\rho_{q}$ moves out of the $\partial \psi / \partial \rho \propto \rho^{-1}$ region; the growth of $j^{*} / j_{\infty}$, as given in (30), is then stopped by the rapid decrease in $\rho_{0} / \tau_{0}$ before the boundary of $A^{*}$ is reached. The effect is the same for all smaller $\beta^{*}$ so that the current is now insensitive to the actual value of $\beta^{*}$. The critical value of $\beta^{*}$ is found by putting $\rho_{q} \approx \rho_{m}$, i.e., $\tau_{q} \approx 1$ leading to $\beta^{*} \propto \epsilon^{2}$; the maximum possible current peak is

$$
\bar{j}^{*} / j_{\infty} \propto \psi_{p}^{1 / 2} \bar{\delta} / \epsilon,
$$

which is roughly proportional to $\left(\epsilon \ln \epsilon^{-1}\right)^{-1}$. This conclusion is supported by the computations of Ref. 1 .

For increasing ion temperatures $\alpha^{*}$ goes up and point $q$ in Fig. 2 moves down, reaching $\rho=1$ at $\alpha^{*}=1$. For $\alpha^{*} \geq 1$, we have $G\left(\rho_{0}\right) / \rho_{0} \leq 1$ for all $\rho_{0}$ so that $\hat{a}^{*}$ never grows quadratically in $\tau$; thus, $\alpha^{*}<1$ may roughly be considered as a necessary condition for the overshoot. The numerical results of Ref. 1 (largest $\left.\alpha^{*} \approx 0.70\right)$ support this prediction too.

To conclude the preceding discussion we note that (a) for $\bar{\rho}_{A} \lesssim \rho_{m}$, the maximum possible error in $\bar{\rho}_{A}(\phi)$, due to equating $\langle\nu\rangle$ to $\bar{\nu}$ is about $1 \%{ }^{6}$ and the one due to equating $\delta$ to $\bar{\delta}$ about $2 \%$; (b) in principle, condition (29) is necessary but not sufficient for $\left(\rho_{0}, \phi_{0}\right)$ to belong to $A^{*}$. For sufficiency, the righthand side of Eq. (27) has to be positive for all $\rho$ between $\rho_{0}$ and 1 ; in other words, no transient potential barriers should appear. It is easy to verify, however, that this more stringent requirement is indeed satisfied for $\rho_{0} \lesssim \rho_{m}$ whenever (29) is. ${ }^{7}$

The quantitative determination of $\bar{\jmath} / j_{\infty}$ involves essentially four steps: (1) Computation of the time of flight to the probe $\tau_{0}\left(\rho_{0}, \phi_{0}\right)$ starting from Eq. (27). (2) Inversion of that expression to find $\rho_{\max }\left(\phi_{0}, \tau\right)$, the maximum $\rho_{0}$ collected at a given $\tau$ for each $\phi_{0}$. (3) Integration over $\left(\rho_{0}, \phi_{0}\right)$, within the bounds $\rho_{0}<$ $\bar{\rho}_{A}(\phi), \rho_{\max }$, to obtain $\hat{a}^{*}$ and therefore $\bar{\jmath}^{*} / j_{\infty}$. (4) Integration over $v_{\perp} *$ using the undisturbed ion distribution function [see Eqs. (8) and (10)]. The derivation is long and tedious; details are given in the Appendix. The final formula is

$$
\begin{gathered}
\bar{J} / j_{\infty}=\left[\pi \epsilon \sigma^{2} /\left(8 \psi_{p}\right)^{1 / 2} \tau\right] X\left(\sigma, \eta, \rho_{m}\right), \\
X=X_{1}+X_{2}-X_{3},
\end{gathered}
$$

where $\rho_{m}, \sigma, \eta$ are given by Eqs. (22),

$$
\begin{gathered}
\sigma \operatorname{erf}(\ln \sigma)^{1 / 2}=\left(2 \psi_{p} \bar{\delta}\right)^{1 / 2} \epsilon^{-1} \tau\left(\pi+\bar{\nu} \bar{g}_{2} \tau^{2}\right)^{-1 / 2}, \\
\eta=2 \beta(\sigma-1) /\left(\psi_{p} \bar{\delta} \ln \sigma^{2}\right) ;
\end{gathered}
$$

$\bar{\nu}$ and $\tilde{\delta}$ are given by Eqs. (25), (24), and Fig. 3;

and

$$
\bar{g}_{2}=0.75 \text {, }
$$

$$
\begin{array}{r}
X_{1}=\operatorname{erf} \eta^{-1 / 2}-2 \eta^{-1 / 2} \operatorname{erfc} \eta^{-1 / 2}+2(\pi \eta)^{-1 / 2} \exp \left(-\eta^{-1}\right) \\
+2 \frac{1-\sigma^{-2}}{\ln \sigma^{2}}\left[\eta^{-1} E_{1}\left(2 \eta^{-1}\right)-2(\pi \eta)^{-1 / 2}\right. \\
\left.\times \exp \left(-\eta^{-1}\right) \operatorname{erfc} \eta^{-1 / 2}\right] \\
X_{2}=\left[\frac{1+4 / \pi}{\pi^{1 / 2}} \frac{\eta^{1 / 2}}{\sigma^{2}}-\frac{2}{3} \frac{\bar{\nu}}{\rho_{m}^{2}} \frac{\sigma^{2}-1}{\ln \sigma^{2}}(\pi \eta)^{-1 / 2}\right] \\
\times \exp \left(-\eta^{-1}\right) \\
X_{3}=\frac{1}{2 \sigma^{2}}+\pi^{-1} \sin ^{-1} \sigma^{-1}+\frac{\left(1-\sigma^{-2}\right)^{1 / 2}}{\pi \sigma} \\
\times\left[1-\left(\frac{2 \eta \ln \sigma^{2}}{\sigma^{2}-1}\right)^{1 / 2}\right]
\end{array}
$$




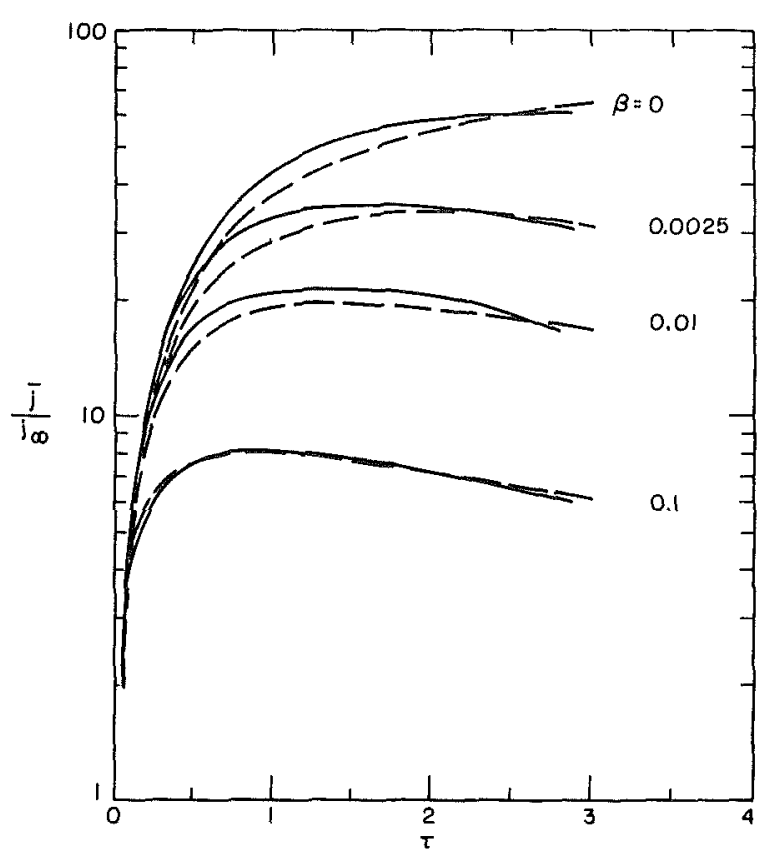

FIG. 5. Time dependence of nondimensional ion current $\vec{\eta} / j_{\infty}$; $\epsilon=10^{-2}, \psi_{p}=15$. Dashed lines, computations of Ref. 1; full lines present theory.

It is shown in the Appendix that $\tau_{0}$ is about 2.80 for $\rho_{0}=\rho_{m}$, so that preceding results will be valid for $\tau<3$. Also, both $\psi_{p}$ and $\epsilon^{-1}$ must be large.

$X$ is given graphically in Fig. 4 for $5 \leq \sigma \leq 5.78 \times 10^{2}$ (this is the largest value of $\sigma$ for the $\epsilon, \psi_{p}$ range mentioned in Ref. 6). We note that (a) for $\sigma>100, X$ is practically independent of $\sigma$, and (b) Fig. 4 does not depend on $\rho_{m}$ : we used an appropriate, intermediate value of $\rho_{n}$ for each curve, and the error for other values of $\rho_{m}$ is less than $\pm 1 \%\left(X_{2}\right.$ is never more than $2 \%$ of $X$ ).

For comparison with the approximate numerical computations of Ref. 1, in Fig. 5 we give $\bar{J} / j_{\infty}$ versus $\tau$ for $\epsilon=10^{-2}, \psi_{p}=15$ and several values of $\beta$, from both those computations and our formula. The agreement is good. ${ }^{8}$

\section{CONCLUSIONS}

We have derived an analytical formula for the transient ion current to a long cylindrical Langmuir probe in a quiescent plasma, assuming that at time $t=0$ a large, negative potential relative to the plasma $\left(-e V_{p} \gg_{\kappa} T_{e}, \kappa T_{i}\right)$ is applied to the probe. We also assumed that the probe was originally at zero potential, that the ion distribution function is Maxwellian, and that the Debye length $\lambda_{D}$ is small compared with all mean free paths and large compared with the probe radius $r_{p}$.

The ion current density $j(t)$ exhibits a large overshoot before approaching its new steady-state value $j_{\infty}$ $\left[j_{\infty}\right.$ is given by Eq. (6)]. This overshoot is sensitive to the ion temperature and may be used for diagnostic purposes. The results of the present theory may be used to this effect. Let $-e V_{p} / \kappa T_{e}=\psi_{p}$ and $r_{p} / \lambda_{D}=\epsilon$ (to be known from conventional steady-state measurements, say) and let

$$
\frac{\bar{j}_{m}}{j_{\infty}} \equiv \tau^{-1} \int_{0}^{\tau} d \tau^{\prime} \frac{j_{m}\left(\tau^{\prime}\right)}{j_{\infty}}
$$

be the nondimensional, time-averaged current density, experimentally measured as a function of $\tau \equiv \omega_{p i} t$, where $\omega_{p i}$ is the ion plasma frequency. Then, a single point in the curve $\vec{\jmath}_{m} / j_{\infty}$ versus $\tau$ can yield the ion temperature: For any couple of values $\left(\bar{j}_{m} / j_{\infty}, \tau\right)$, the quantities $\sigma$ and $X$, as defined by Eqs. (35) and

$$
X=\left(\bar{\jmath}_{m} / j_{\infty}\right)\left(8 \psi_{p}\right)^{1 / 2}\left(\pi \epsilon \sigma^{2}\right)^{-1} \tau,
$$

can be evaluated; then $\eta(\sigma, X)$ can be obtained from Fig. 4 , and the temperature ratio follows from

$$
\beta \equiv T_{i} / Z_{i} T_{s}=\eta \psi_{p} \bar{\delta} \ln \sigma^{2} / 2\left(\sigma^{2}-1\right) .
$$

In these equations $\bar{\delta}$ is defined by Eq. (24), where $Y\left(\epsilon, \psi_{p}\right)$ is given by Fig. 3, and $\vec{\nu}$ and $\bar{g}_{2}$ are given by Eqs. (25) and (37). The value of $\tau$ chosen should optimally, be between 2 and 2.5 (if $\tau$ is too small, $X$ may be too close to unity to determine $\eta$ from Fig. 4 , while for $\tau>3$ our analysis breaks down).

While deriving an analytical formula for $\bar{\jmath}_{m} / j_{\infty}$ [given in Eq. (33) and below it], certain functions $\delta$, $\langle\nu\rangle$, and $g_{2}$, which vary between narrow bounds, were approximated inside a number of integrals by the constants $\bar{\delta}$ (in time), $\vec{\nu}$, and $\bar{g}_{2}$. These approximations produce errors in the determination of $T_{i}$, that follow easily from Eqs. (33) to (42). For large $\sigma$, we can write $T_{i} \propto\left(\pi+\vec{g}_{2} \bar{\nu} \tau^{2}\right) \eta[X(\sigma)]$, and $\sigma \propto \bar{\delta}^{1 / 2}(\pi+$ $\left.\bar{g}_{2} \bar{\nu} \tau^{2}\right)^{-1 / 2}$, while for $2<\eta<30$ we roughly have $X \approx 1-$ $0.20 \ln \eta$. From the bounds of $\delta$ found in Sec. III we get an upper estimate for the possible error due to our equating $\delta$ to $\bar{\delta}$, of less than $10 \% .{ }^{6}$ At $\tau=1.5$ we get a similar result for the product $\bar{g}_{2} \bar{\nu}$. We would like to stress that these are very conservative estimates and that the actual error in $T_{i}$ should not be larger than a few percent.

The error due to $\vec{g}_{2} \bar{p}$ grows with $\tau$; our estimate for it reaches $20 \%$ at $\tau=2.80$. Again, this is a very conservative estimate, but clearly indicates that the theory should not be used, except for gross predictions, for $\tau$ larger than, say, 3. It should be pointed out that there is also a lower bound for $\tau$ (say $r=0.1$ ) which arises from the use of the Boltzmann law for the electron density $[\mathrm{Eq} .(5)] .^{3}$

The restricted range $0.1 \lesssim \tau \lesssim 3$ creates an experimental limitation. To resolve time on the ion plasma frequency scale it is necessary that the time constant $R C$ of the measuring device be very small compared with the ion plasma period, $2 \pi / \omega_{p i}$. Assuming that the resistance $R$ is governed by the requirement that the probe current give rise to a potential $V=v \times 10^{-3} \mathrm{~V}$ across it, and that the capacitance of the circuit is 
$C=c \times 10^{-12} \mathrm{~F}$, we obtain the condition

$$
v c \ll 3 \times 10^{-3} \psi_{p}^{1 / 2} \epsilon\left(l / \lambda_{D}\right) N_{0} \lambda_{D}{ }^{3}\left(j_{m} / j_{\infty}\right),
$$

where $l$ is the length of the probe. For typical instrumentation, $v$ should not be less than about unity, and $c$ would be larger than unity, but of that order. We may use $\bar{\jmath} / j_{\infty}$ from Eq. (33) for $j_{m} / j_{\infty}$ in (43), with $X$ between 0.2 and unity; then, typically we get the condition $\psi_{p}\left(N_{0} \lambda_{D}{ }^{3}\right) l / \lambda_{D} \gg 10^{5}$.

An important point to consider is whether the current measured (that is the current in the circuit outside the plasma) can really be identified with the ion current as we have done until now. The equation for probe charge conservation is

$$
j_{D} \equiv \frac{\partial q_{s}}{\partial t}=j-j_{e}-j_{m},
$$

where $q_{s}$ is the surface charge density, $j$ and $j_{e}$ are the ion and electron plasma current densities, and $2 \pi r_{p} l j_{m}$ is the current measured experimentally; $j_{e}$ may be neglected because it is exponentially small in $\psi_{p}$ so that our method will be useful as long as $j_{D} / j$ is small. The displacement current $j_{D}$ is due to the rearrangement in space-charge density following the application of the potential $V_{p}$. We have $j_{D}=(4 \pi)^{-1}$ $(\partial / \partial t)(-\partial V / \partial r)_{r=r_{p}} \equiv V_{p}\left(4 \pi r_{p}\right)^{-1} \partial \delta / \partial t$, where the function $\delta(\tau)$ was studied in Sec. III. Time averaging we find

$$
\bar{\jmath}_{D} / j_{\infty}=\pi \psi_{p}^{1 / 2}[\delta(\tau)-\delta(\tau \approx 0)] / 2^{1 / 2} \epsilon \tau .
$$

Notice that we averaged $j_{D}$ between $\tau \approx 0$ (for numerical purposes $\tau=0.1)^{3}$ and $\tau$. The average of $j_{m}$ to be used in Eq. (41) should be understood in the same sense. It is convenient to define this kind of average because only for very small $\tau(\tau<0.1)$ will $j_{D}$ be large.

A conservative estimate of $[\delta(\tau)-\delta(\tau \approx 0)] \tau^{-1}$, would appear to be $[\delta(\tau \rightarrow \infty)-\delta(\tau \approx 0)](2 \pi)^{-1}$ because the calculations of Ref. 1 indicate that the asymptotic approach to $\delta(\tau \rightarrow \infty)$ requires several ion plasma periods. Then, we find that $\vec{\jmath}_{D} / j_{\infty}$ is just less than unity for $\epsilon^{-1}=\psi_{p}=10^{2}$, and decreases with both $\psi_{p}$ and $\epsilon^{-1}$. Since $\bar{J} / j_{\infty}$ is typically of order of $10^{2}$ for such $\epsilon$ and $\psi_{p}$, and has roughly the same dependence on $\epsilon$ and $\psi_{p}$ that $\vec{\jmath}_{D} / j_{\infty}$ has, it appears that $\vec{\jmath}_{D} / \vec{\jmath}$ will always be of order of $1 \%$. Therefore, in any actual experiment $j_{m}$ will show a big peak due to $j_{D}$, that will die off very rapidly $(\tau<0.1)$. After that, a second peak, due to $j$, will appear; this is the "overshoot" of interest here.

An alternative way of measuring $T_{i}$ would be to apply a potential pulse of width $3 / \omega_{p i}$ (instead of the stepwise potential discussed so far) to the probe. If the time average now extends from a few electron plasma periods before the beginning of the pulse up to a few electron plasma periods after the pulse is ended, it is easy to verify that the displacement current averages to zero.
The requirement that the probe potential for $r<0$ (call it $V_{p}^{-}$) be zero, only means, of course, that $V_{p}-$ must be small in some sense, since the plasma potential can never be known exactly. To clarify this point, let us consider a series of measurements for different values of $\psi_{p}^{-} \equiv-e V_{p}^{-} / \kappa T_{e}$, and let us call $\bar{\jmath} / j_{\infty} \equiv I\left(\psi_{p}^{-}\right)$so that $I(0)$ is given by Eq. (33). From steady-state theory ${ }^{2}$ one knows $n_{i}^{-}$and $n_{e}^{-}$as functionals of $\psi^{-}\left(n_{i}^{-}, n_{e}^{-}\right.$, and $\psi^{-} \equiv-e^{-} / T_{e}$ being the ion and electron densities, and the potential field, for $\tau<0$ ) so that it is possible to determine the gross behavior of $\psi^{-}\left(\rho, \psi_{p}, \beta, \epsilon\right)$. We then find that $I \approx I(0)$ for $\psi_{p}^{-}$within a certain neighborhood of zero, while for $\psi_{p}^{-}$outside it, $I$ decreases as $\left|\psi_{p}-\right|$ increases. Thus, $I$ passes through a maximum at $\psi_{p}-=0$. The reason why $I$ decreases for $\psi_{p}^{-}$positive and larger than some value is that the angular momentum of the ions around the probe, at $\tau<0$, is substantially increased by the field $\psi^{-}$, so that the boundary of $A^{*}$ in Fig. 2 moves down. This effect depends on $\beta$ so that the width of the maximum of $I$ for $\psi_{p}->0$ decreases with $\beta$. On the other hand, the decrease of $I$ for $\psi_{p}{ }^{-}<0$ is due to the depletion of ions caused by the repelling field $\psi^{-}$; the penetration of this field inside the plasma is governed by the electrons so that it does not depend on $\beta$. Thus, the width of the maximum of $I$ for $\psi_{p}{ }^{-}<0$, for small $\beta$, is essentially independent of $\beta$ : as $\beta \rightarrow 0$ one need not use a vanishingly small $\psi_{p}{ }^{-}$. We can roughly state that $I\left(-\psi_{p}-<0.1\right) \approx$ $I(0)$ for $\beta \ll 1$ and $\tau \gg 0.1$.

In a real situation, then, the way to proceed is as follows. Both the floating potential (relative to an arbitrary origin) and $T_{e}$ will be assumed to be known, so that the plasma potential can be determined, within about $\pm \kappa T_{e} / e$ vs (typically $-e V_{f} / \kappa T_{e} \approx 4-6$ ). One should then obtain a steady state at the most negative potential of this range, measure $j_{m}(t)$ after the application of the potential $V_{p}$, and repeat this procedure at intervals of, $\Delta V_{p}{ }^{-}=+0.1 \kappa T_{e} / e$, until a turning point (maximum) of $I$ is reached. If the maximum of $I$ is reached at only one station $V_{p *}{ }^{-}$, it may be convenient to repeat the procedure around $V_{p *^{-}}$using a smaller value of $\Delta V_{p}^{-}$. Once $I_{\max }$ has been found, and only then, one should apply the algorithm for the determination of $T_{i}$ that was indicated at the beginning of this section. We conclude that the sensitivity of $I$ to $\psi_{p}-$ is not a drawback of the method since the plasma potential can be determined along with $T_{i}$.

For a given probe radius and plasma density, there is a natural lower bound to the range of ion temperatures that can be measured with this method. ${ }^{5}$ Note that $T_{i}$ can be determined only if $\eta>1$, that is,

$$
\eta \approx\left(\beta / \epsilon^{2}\right)\left(4 / \ln \sigma^{2}\right)\left[\tau^{2} /\left(\pi+\bar{\nu} \bar{g}_{2} \tau^{2}\right)\right]>1
$$

which means that the ion Debye length must be larger than the probe radius; (the minimum $\beta$ is 
about $2 \times 10^{-4}$ for $\epsilon=10^{-2}$, about $10^{-2}$ for $\epsilon=10^{-1}$, and slightly less than $10^{-1}$ for $\epsilon=10^{-1 / 2}$ ). If the value of $X$ found from $\mathrm{Eq} .(41)$ is less than unity, one can determine $\eta$ and then $T_{i}$; if, on the other hand, $X$ is very close to unity, one can only say that $\eta \leq 1$, and, therefore, all that can be determined is an upper limit to $T_{i}$.

\section{ACKNOWLEDGMENTS}

The author is highly grateful to Professor A. A. Sonin for helpful discussions. The author also acknowledges valuable criticism from the referee.

This research was supported by the Advanced Research Projects Agency of the Department of Defense and was monitored by the Office of Naval Research under Contract No. N00014-0204-0040, ARPA Order No. 322.

\section{APPENDIX}

For $\phi_{0} \leq \pi / 2$, the time of flight to the probe is

$$
\tau_{0}=\int_{1}^{\rho_{0}} d \rho\left[\left(\frac{d \rho}{d \tau}\right)^{2}\right]^{-1 / 2}
$$

where $\left(d_{\rho} / d \tau\right)^{2}$ is given by Eq. (27). Expanding $(d \rho / d \tau)^{2}$ around $2 \epsilon^{-2}\left[-\psi_{p} \bar{\delta} \ln \left(\rho / \rho_{0}\right)+\beta^{*} \cos ^{2} \phi_{0}\right]$ we find

$\tau_{0} \approx \pi^{1 / 2} \frac{\rho_{0}}{\rho_{m}} \exp \left(\frac{\alpha_{c}^{*}}{2}\right) \frac{\operatorname{erf}\left(\ln \rho_{0}+\alpha_{c}^{*} / 2\right)^{1 / 2}-\operatorname{erf}\left(\alpha_{0}^{*} / 2\right)^{1 / 2}}{\left[1-\alpha^{*} \sin ^{2} \phi_{0}-\bar{\nu} g_{1}\left(\alpha_{0}^{*}\right) \rho_{0}^{2} / \rho_{m}^{2}\right]^{1 / 2}}$

with an error of (at most) a few percent; $\alpha_{0}{ }^{*}=\alpha^{*} \cos ^{2} \phi_{0}$ and $g_{1}(y) \equiv 3^{1 / 2} e^{2}\left[\left(\operatorname{erfc}(3 y / 2)^{1 / 2} / \operatorname{erfc}(y / 2)^{1 / 2}\right)-1\right]$. For $\phi_{0}>\pi / 2$, we get

$$
\begin{aligned}
\tau_{0} \approx \tau_{0}\left(\pi-\phi_{0}\right)+ & \frac{\left(8 \alpha_{c}^{*}\right)^{1 / 2} \rho_{0} / \rho_{m}}{1-\alpha^{*}-\bar{\nu} \rho_{0}{ }^{2} / \rho_{m}{ }^{2}} \\
& \times\left[1-\frac{2 \alpha_{c}^{*}\left(1-2 \bar{\nu} \rho_{0}{ }^{2} / \rho_{m}{ }^{2}\right)}{3\left(1-\alpha_{0}{ }^{*}-\bar{\nu} \rho_{0}{ }^{2} / \rho_{m}{ }^{2}\right)^{2}}\right]
\end{aligned}
$$

the second term comes from the range $\rho>\rho_{0}$, where $(d \rho / d \tau)^{2}$ can be approximated by a polynomial of second degree in $\rho$.

From Eq. (A1) we get

$$
\begin{aligned}
& \rho_{0}^{2}\left[\operatorname{erf}\left(\ln \rho_{0}+\alpha_{o}^{*} / 2\right)^{1 / 2}-\operatorname{erf}\left(\alpha_{0}^{*} / 2\right)^{1 / 2}\right] \\
& \approx \rho_{m}{ }^{2} \tau_{0}{ }^{2}\left(1-\alpha^{*}\right) /\left(\pi+\vec{g}_{2} \bar{\nu} \tau^{2}\right)
\end{aligned}
$$

by approximating $\exp \left(-\alpha_{0}^{*}\right)\left(1-\alpha^{*} \quad \sin ^{2} \phi_{0}\right)$ by $1-\alpha^{*}$ (error of less than $1 \%$ for $\alpha^{*}<0.1$ ), and the function $g_{2}\left(\alpha_{0}^{*}\right) \equiv g_{1}\left(\alpha_{c}^{*}\right) \exp \left(-\alpha_{c}^{*}\right) \operatorname{erfc}\left(\alpha_{0}^{*} / 2\right)$ by $\bar{g}_{2} \equiv 0.75\left[g_{2}(y)\right.$ varies between 0.732 and 0.782 for $0 \leq y \leq 0.1]$. We also approximated $1+\pi^{-1} \bar{\nu} \bar{g}_{2} \tau^{2}[1-$ $\left.\operatorname{erfc}\left(\ln \rho_{0}+\alpha_{o}{ }^{*} / 2\right)^{1 / 2} / \operatorname{erfc}\left(\alpha_{c}^{*} / 2\right)^{1 / 2}\right]^{-2}$ by $1+\pi^{-1} \bar{\nu} \bar{g}_{2} \tau^{2}$; this follows from the fact that $\rho_{m}{ }^{2}$ is very large. If one neglects some terms of (A2) that contribute less than $3 \%$ of $\rho_{0}{ }^{2}\left(\phi_{0}>\pi / 2\right)$ (and therefore little more than $1 \%$ of $\left.\hat{a}^{*}\right)$, one finds that Eq. (A3) also holds for $\phi_{0}>\pi / 2$, provided that now the erf functions inside the bracket add to each other.

We next expand $\operatorname{erfc}\left(\alpha_{0}^{*} / 2\right)^{1 / 2}$ for small $\alpha_{c}^{*}$ to within $1 \%$ and write

$\operatorname{erf}\left(\ln \rho_{0}+\alpha_{c}^{*} / 2\right)^{1 / 2} \mp \operatorname{erf}\left(\alpha_{c}^{*} / 2\right)^{1 / 2}$

$$
\begin{gathered}
\approx\left[1-\left(2 \alpha^{*} / \pi\right)^{1 / 2} \cos \phi_{0}\right] \\
\times\left[1-\operatorname{erfc}\left(\ln \rho_{0}+\alpha_{0}^{*} / 2\right)^{1 / 2} /\left\{1-\left(2 \alpha^{*} / \pi\right)^{1 / 2} \cos \phi_{0}\right\}\right] .
\end{gathered}
$$

We approximate the second bracket as

$$
1-\operatorname{erfc}\left(\ln \left\{\rho_{0}\left[1-\left(2 \alpha^{*} / \pi\right)^{1 / 2} \cos \phi_{0}\right]\left(1-\alpha^{*}\right)^{-1 / 2}\right\}\right)^{1 / 2} \text {. }
$$

This is exact for either $\alpha^{*} \rightarrow 0$ or $\rho_{0} \rightarrow \infty$. Otherwise, there is an error that vanishes at some $\phi_{0}$ between 0 and $\pi$, and has opposite signs for larger and smaller angles; this leads to a partial error balance. For $\tau_{0}>0.1$ (so that $\rho_{0}$ is large) and $\alpha^{*}<0.1$ the error is at most about a few percent. Finally, we obtain

$$
p_{\max }{ }^{2}=\sigma^{2}\left(1-\alpha^{*}\right)\left[1-\left(2 \alpha^{*} / \pi\right)^{1 / 2} \cos \phi_{0}\right]^{-2},
$$

where $\sigma$ is given by $\mathrm{Eq}$. (35). We have written $\rho_{\max }$ for $\rho_{0}$ and $\tau$ for $\tau_{0}$; Eqs. (A4) and (35) now represent the maximum $\rho_{0}$ collected at time $\tau$ for a given $\phi_{0}$. The dependence of Eq. (A4) on $\phi_{0}$ and $\alpha^{*}$ is remarkably simple. Note that condition $\rho_{0} \lesssim \rho_{m}$ together with Eqs. (A4) and (35) lead to the more useful condition $\tau<\tau_{m} \approx 2.80$

To obtain $\hat{a}^{*}(\tau)$ we integrate over $\rho_{0}$ up to either $\rho_{\max }\left(\phi_{0}, \tau\right)$ or $\bar{\rho}_{A}\left(\phi_{0}\right)$, whichever is the smallest, and then integrate over $\phi_{0}$. We again schematically drew the boundaries of $A^{*}$ in Fig. 6. We also included several curves of the $\rho_{\max }\left[\phi_{0}, \sigma(\tau)\right]=$ constant family. Notice that there is a value $\sigma_{L}$ such that if $\sigma<\sigma_{L}$, then $\rho_{\max }\left(\phi_{0}, \sigma\right)$ and $\bar{\rho}_{A}\left(\phi_{0}\right)$ do not intersect; for $\sigma>\sigma_{L}$ there are two intersection points, at angles $\phi_{1}(\sigma)$ and $\phi_{2}(\sigma)$. At $\sigma=\sigma_{L}$, we have $\phi_{1}=\phi_{2} ; \sigma_{L}$ is given by $\alpha^{*}=h\left(\sigma_{L}\right)$, where

$$
h(\sigma)=\left[\frac{\ln \sigma^{2}}{\sigma^{2}}-\frac{2}{\pi}\left(\frac{\ln \sigma^{2}-1}{\sigma^{2}}\right)^{2}-\frac{\bar{\nu}}{\rho_{m}^{2}}\right]\left(1-\frac{\ln \sigma^{2}}{\sigma^{2}}\right)^{-1} .
$$

If we write $\hat{a}^{*}=\hat{a}_{1}{ }^{*}-\hat{a}_{2}{ }^{*}$, where $\hat{a}_{2}{ }^{*}$ corresponds to the area of the probe and of that part of the shaded region that lies inside the curve $\rho_{0}=\rho_{\max }\left(\phi_{0}, \sigma\right)$, we get

$$
\hat{a}_{1}^{*} \approx \pi \sigma^{2}
$$

$$
a_{2}^{*} \approx \frac{1}{2} \pi+\sigma^{2} \sin ^{-1} \sigma^{-1}+\left(\sigma^{2}-1\right)^{1 / 2}-2 \sigma\left(2 \alpha^{*} / \pi\right)^{1 / 2},
$$

for $\tau<\tau_{L} \equiv \tau\left(\sigma_{L}\right)$, with an error of less than $1 \%$. For $\tau>\tau_{L}, \hat{a}_{2}{ }^{*}$ is still given by (A5) but for $\hat{a}_{1}^{*}$ we now have

$$
\begin{aligned}
a_{1}^{*}=\int_{0}^{\phi_{1}(\sigma)} \rho_{\max }{ }^{2}\left(\phi_{0}, \sigma\right) d \phi_{0} & +\int_{\phi_{1}(\sigma)}^{\phi_{2}(\sigma)} \bar{\rho}_{A}{ }^{2}\left(\phi_{0}\right) d \phi_{0} \\
& +\int_{\phi_{2}(\sigma)}^{\pi} \rho_{\max }{ }^{2}\left(\phi_{0}, \sigma\right) d \phi_{0} .
\end{aligned}
$$


Fig, 6. Diagram for the determination of $\hat{a}^{*}(\tau)$.

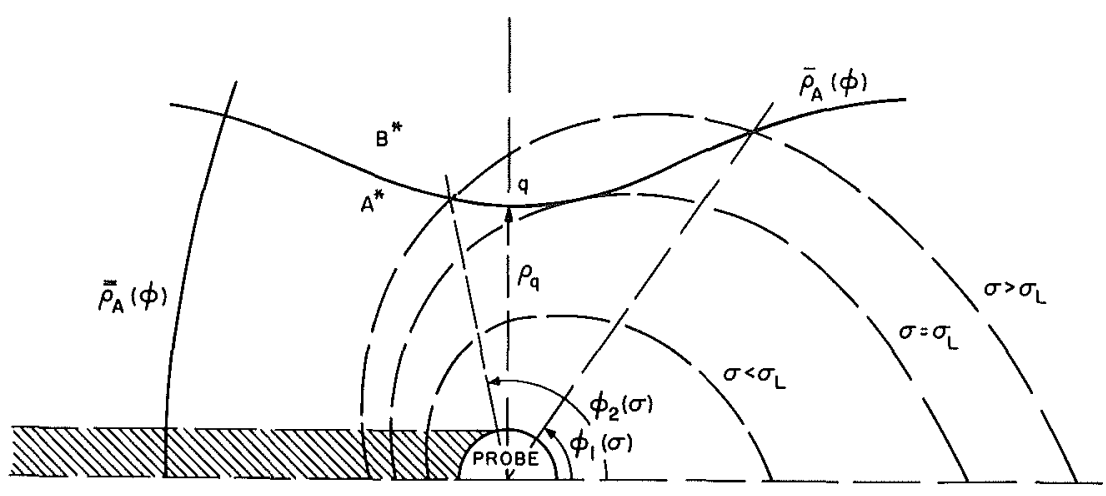

The first and third integrals can easily be carried out. To calculate the second integral we approximate $\bar{\rho}_{A}{ }^{2}\left(\phi_{0}\right)$ from Eq. (29) by

$$
\begin{aligned}
\bar{\rho}_{A}{ }^{2} \approx & \left(\alpha^{*} \sin ^{2} \phi_{0}+\bar{\nu} / \rho_{m}^{2}\right)^{-1} \ln \left[1.32 \rho_{q}^{2}\right. \\
& \left.\times \exp \alpha^{*}\left(\alpha^{*}+\bar{\nu} / \rho_{m}^{2}\right)\left(\alpha^{*} \sin ^{2} \phi_{0}+\bar{\nu} / \rho_{m}{ }^{2}\right)^{-1}\right] .
\end{aligned}
$$

The maximum error in this approximation is $7.7 \%$ at $\alpha^{*}=0.1$ for a certain value of $\phi$; obviously

$$
\int_{\phi_{1}}^{\phi_{2}} \bar{\rho}_{A^{2}} d \phi_{0}
$$

will be more accurate [the more so since the error in Eq. (A7) can have either sign]. Moreover, (1) after the integration is performed we again use the exact form for $\bar{\rho}_{A}$, so that the error is reduced, and (2) the error is a maximum when the contribution of

$$
\int_{\phi_{1}}^{\phi_{2}} \bar{\rho}_{A}^{2} d \phi_{0}
$$

to $a^{*}$ is minimum. The error due to the use of Eq. (A7) should be, at most, a few percent. The final expression for $\hat{a}_{1}{ }^{*}\left(\tau>\tau_{L}\right)$ is long and complicated.

From Eqs. (8), (10), and (11) it follows that

$\frac{\bar{\jmath}}{j_{\infty}} \equiv \tau^{-1} \int_{0}^{\tau} \frac{j\left(\tau^{\prime}\right)}{j_{\infty}} d \tau^{\prime}=\frac{\epsilon}{\left(8 \psi_{p}\right)^{1 / 2} \tau} \int_{0}^{\infty} \frac{d \alpha^{*}}{\alpha} \exp \left(-\frac{\alpha^{*}}{\alpha}\right) \hat{a}^{*}$,

where $\alpha^{*} / \alpha=\beta^{*} / \beta=m_{i}\left(v_{\perp}\right)^{2} / 2 \kappa T_{i}$. The integral of $\hat{a}_{2} *$ can easily be carried out and leads to the $X_{3}$ term in
Eq. (34). In the integral of $\hat{a}_{1}^{*}, \mathrm{Eq}$. (A5) must be used for $\alpha^{*}<h(\sigma)$ and $\mathrm{Eq}$. (A6) for $\alpha^{*}>h(\sigma)$. After considerable simplification one obtains the $X_{1}$ and $X_{2}$ terms of Eq. (34) with an error of less than $1 \%$.

${ }^{1}$ S. D. Hester and A. A. Sonin, Phys. Fluids 13, 1265 (1970). $2 \mathrm{~J}$. G. Laframboise, University of Toronto Institute for Aerospace Studies Report No. 100 (1966); see Appendix E for steady. state densities and currents, for a cylindrical probe drawing orbital-motion limited current. For additional references see F. F. Chen, in Plasma Diagnostic Techniques, edited by R. H. Huddlestone and S. L. Leonard (Academic, New York, 1965), Chap. 4.

${ }_{3} B y=\tau \approx 0$ we mean a time $\tau \ll 1$ such that $\mathrm{Eq} .(5)$ is already satisfied, while the ions have had no time to respond to the field. As indicated below Eq. (5), $r=0.1$ would be a valid choice.

${ }^{4}$ It will suffice to note that $\psi\left(\tau \rightarrow \infty, \rho>\rho_{(3)}\right)>p \rho^{2}(3) / \rho^{2}$, as follows from the condition for orbital-motion-limited current collection.

${ }^{5}$ Since $\rho_{m}$ grows with $\epsilon^{-1}$, it is clear that the smaller the probe, the smaller the minimum temperature that can be measured. On the other hand, note that although $\rho_{m}$ grows with $\psi_{p}$, so does $\rho_{q}$ : a change in the probe potential has no effect on the range of temperature that can be measured.

${ }^{6}$ Whenever we needed numerical estimates of errors due to simplification of the analysis arising from conditions $\psi \gg 1$, $\epsilon \ll 1, \alpha \ll 1$, we specifically assumed $10 \leq \psi_{p} \leq 10^{2}, 10^{-2} \leq \epsilon \leq 10^{-1}$, $\alpha<10^{-1}$.

${ }^{7}$ For $\phi_{0}>\frac{1}{2} \pi$, there is the obvious additional requirement that the right-hand side of Eq. (27) be zero at some $\rho>\rho_{0}$. This other boundary of $A, \overline{\bar{\rho}}_{A}(\phi)$, is represented in Fig. 2 too; it plays no role in our analysis because $\overline{\bar{\rho}}_{A}$ is clearly larger than $\rho_{m}$.

${ }^{8}$ Indirect experimental evidence of the correctness of our analysis is provided by some current data for probes in rarefied plasma flows: R. T. Bettinger and A. A. Chen, J. Geophys. Res. 73, 2513 (1968) and Ref. 1. See J. R. Sanmartin, Phys. Fluids (to be published) and Ref. 1 for a theory on the similarity between the plasma flow problem and the present one. 